\title{
ERRATUM
}

\section{Heavy Metal Concentrations in Feathers of Korean Shorebirds}

\author{
Jungsoo Kim · Tae-Hoe Koo
}

Published online: 4 March 2008

(C) Springer Science+Business Media, LLC 2008

\section{Erratum to: Arch Environ Contam Toxicol (2008) \\ DOI 10.1007/s00244-007-9089-y}

In the online and printed version of this article, the $p$-values

in Table 3 are incorrect. They appear correctly below:

Table 3 Heavy metal concentrations (geomean, 95\% CI $\mu \mathrm{g} / \mathrm{g}$ wet weight) in the liver of shorebirds from Yeongjong Island, Korea

\begin{tabular}{|c|c|c|c|c|c|c|}
\hline Species & & Zinc & Manganese & Copper & Lead & Cadmium \\
\hline \multirow[t]{2}{*}{ Kentish Plover $(n=5)$} & Mean & 26.6 & 1.35 & 5.51 & $4.26 \mathrm{a}$ & 0.55 \\
\hline & Extreme & $10.6-42.6$ & $0.01-3.02$ & $1.83-9.20$ & $1.19-7.34$ & $0.35-0.75$ \\
\hline \multirow[t]{2}{*}{ Mongolian Plover ${ }^{\mathrm{a}}(n=2)$} & Mean & 30.9 & 1.07 & 5.34 & 2.05 & 0.14 \\
\hline & Extreme & $23.9-38.0$ & $0.01-3.44$ & $3.58-7.12$ & ND-2.05 & $0.01-0.36$ \\
\hline \multirow[t]{2}{*}{ Dunlin $(n=6)$} & Mean & 39.5 & 3.05 & 8.64 & $3.64 \mathrm{a}$ & 0.67 \\
\hline & Extreme & $35.4-43.6$ & $2.43-3.66$ & $1.35-15.9$ & $2.78-4.49$ & $0.46-0.88$ \\
\hline \multirow[t]{2}{*}{ Great Knot $(n=10)$} & Mean & 31.6 & 1.11 & 3.52 & $3.37 \mathrm{a}$ & 0.48 \\
\hline & Extreme & $27.3-35.9$ & $0.65-1.56$ & $0.01-9.87$ & $1.39-5.35$ & $0.13-0.83$ \\
\hline \multirow[t]{2}{*}{ Terek Sandpiper $(n=10)$} & Mean & 20.6 & 1.61 & 6.36 & $0.85 b$ & 0.34 \\
\hline & Extreme & $13.5-27.8$ & $0.96-2.26$ & $2.19-10.5$ & $0.20-1.51$ & $0.14-0.54$ \\
\hline$p$-Value & & NS & NS & NS & 0.001 & NS \\
\hline
\end{tabular}

Note: Means sharing same letter were not significantly different among species. ND $=$ not detected, NS $=$ not significant

${ }^{\text {a }}$ The Mongolian Plover was excluded from statistical analyses because of the small sample size

The online version of the original article can be found under doi: 10.1007/s00244-007-9089-y.

J. Kim · T.-H. Koo $(\bowtie)$

Department of Environmental Science and Engineering,

Kyung Hee University, Yongin, Gyeonggi-do 446-701,

Republic of Korea

e-mail: thkoo@khu.ac.kr 